\title{
CORRIGENDUM
}

\section{Emerging roles of ATF2 and the dynamic AP1 network in cancer}

Pablo Lopez-Bergami, Eric Lau and Ze'ev Ronai

Nature Reviews Cancer 10, 65-76(2010)

On page 68 of this article, the sentence "Consistently, loss of JUN decreases the incidence of papilloma outgrowth by abrogation of epidermal growth factor receptor (EGFR) signalling in skin subjected to the two-stage skin carcinogenesis protocol ${ }^{95}$. ", is incorrectly phrased and referenced. This sentence should read: "Consistently, expression of TAM67 decreases the incidence of papilloma outgrowth from skin subjected to the two-stage skin carcinogenesis protocol ${ }^{128,129}$. Notably, JUN has been shown to contribute to tumour volume - loss of JUN expression in mice that are prone to epidermal growth factor receptor (EGFR)-dependent skin tumour formation results in the same number of tumours, but they are reduced in size ${ }^{95}$." In addition, in Box 2, the sentence "Whereas CRE-BP2 lacks exons 1-7 and most of exon 12, CRE-BP2 varies from CRE-BP1..." should have read "Whereas CRE-BP2 lacks exons 1-7 and most of exon 12, CRE-BP3 varies from CRE-BP1... 